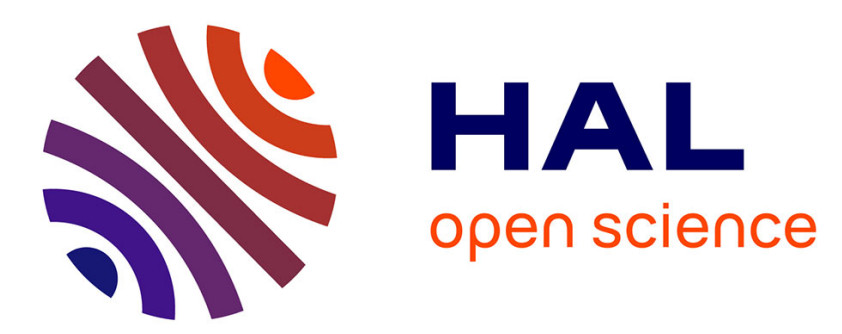

\title{
Phase resolved cross-correlation spectroscopy on surface barrier discharges in air at atmospheric pressure
}

\author{
R. Brandenburg, H. Grosch, T. Hoder, K.-D. Weltmann
}

\section{To cite this version:}

R. Brandenburg, H. Grosch, T. Hoder, K.-D. Weltmann. Phase resolved cross-correlation spectroscopy on surface barrier discharges in air at atmospheric pressure. European Physical Journal: Applied Physics, 2011, 55 (1), 10.1051/epjap/2011100481 . hal-00719805

\section{HAL Id: hal-00719805 \\ https://hal.science/hal-00719805}

Submitted on 21 Jul 2012

HAL is a multi-disciplinary open access archive for the deposit and dissemination of scientific research documents, whether they are published or not. The documents may come from teaching and research institutions in France or abroad, or from public or private research centers.
L'archive ouverte pluridisciplinaire $\mathbf{H A L}$, est destinée au dépôt et à la diffusion de documents scientifiques de niveau recherche, publiés ou non, émanant des établissements d'enseignement et de recherche français ou étrangers, des laboratoires publics ou privés. 


\title{
Phase resolved cross-correlation spectroscopy on surface barrier discharges in air at atmospheric pressure
}

\author{
R. Brandenburg, H. Grosch, T. Hoder, and K.-D. Weltmann \\ Leibniz Institute for Plasma Science and Technology, Felix-Hausdorff-Str. 2, 17489 Greifswald, Germany
}

Received: 03.12.2010 / Revised version: 18.04.2011

\begin{abstract}
Microdischarges in a surface barrier discharge with special asymmetric needle-needle arrangement were investigated by means of cross-correlation spectroscopy (with sub-ns and sub-mm resolution) and an intensified CCD camera equipped with a far field microscope. The surface barrier discharge was driven at conditions (overvoltage) resulting in several microdischarges per half period of the applied sinousoidal voltage. At this conditions and in this arrangement regular patterns of microdischarges between the two electrodes points are formed due to local charging of the dielectric surface. The cross-correlation spectroscopy setup enables the recording of microdischarge development for different phases of the applied voltage. Distinct differences in the microdischarge development between the subsequent phase channels in the positive half period can be observed, while the first channel shows similar behaviour as in the single-microdischarge-mode which was conducted recently.
\end{abstract}

PACS. 52.50.Kz Plasma sources - 52.70.Kz Optical (ultraviolet, visible, infrared) measurements 52.80.Tn Other discharges

\section{Introduction}

Surface barrier discharges (SBDs) in air at atmospheric pressure are typical examples of so-called filamentary plasmas. They consist of erratic appearing, constricted microdischarges (MDs) with short duration, visible as distinct filaments $[1,2]$. Recently the method of cross-correlation spectroscopy (CCS) was successfully applied on surface barrier microdischarges generated in a special asymmetric point-to-point SBD configuration [3]. CCS enables the spectrally resolved study of MD luminosity with high temporal (sub-ns) and spatial (sub-mm) resolution $[4,5]$. In the CCS studies undertaken for barrier discharges so far (volume and coplanar barrier discharges, short VBD and CBD, respectively) the plasma was driven sinusoidal with voltage amplitudes closed to the burning voltage [6-8]. In this situation only one or at most two MDs per half period are generated (so-called single-MD-mode). However, in almost all applications barrier discharges will be driven at voltage amplitudes larger than the burning voltage and thus with a large number of MDs within the same half period.

The method of CCS is not limited to the single-MD mode with localized MDs, only. It offers a phase resolution, i.e. the development of MDs at different phases within the period of applied voltage can be studied. In case of sinusoidal driven VBDs the MD development in the volume (consisting of Townsend-prephase, cathode directed streamer, anode glow and decay phase) was found

$\overline{\text { Send offprint requests to: }}$ brandenburg@inp-greifswald.de to be independent on the phase [6]. However, the MD development and distribution on the surface of the dielectric in all barrier discharges (VBD, CBD, SBD and so called sliding discharges) are influenced by residual charges [912]. On SBDs we found a similar behavior, which will be investigated in this contribution more detailed. Therefore, phase resolved analysis of MDs generated in the positive half period of the applied voltage is made for the same SBD arrangement. The discharge is driven in the singleMD mode (at burning voltage) as well as at over voltage resulting in several MDs per half period of the applied voltage. The aim of the study is to get a more detailed insight of MD development in a more realistic operation mode. In particular the role of the voltage amplitude and residual surface charges will be discussed.

\section{Experimental set-up}

The experimental set-up was already described in [3] and details on the method of CCS can be found elsewhere (see $[4,6]$ and references therein). Here, only the main important facts will be given.

The discharge was generated in an arrangement consisting of two needle electrodes (syringe hollow needles made of chrome-nickel-steel alloy) placed on the opposite sites of a $0.6 \mathrm{~mm}$ thick alumina plate (see Fig. 1). The tips of the electrode faced each other with a gap of $\mathrm{d}=1.15 \mathrm{~mm}$. To prevent parasitic discharges, one side was covered in silicone glue (covered or encapsulated elec- 


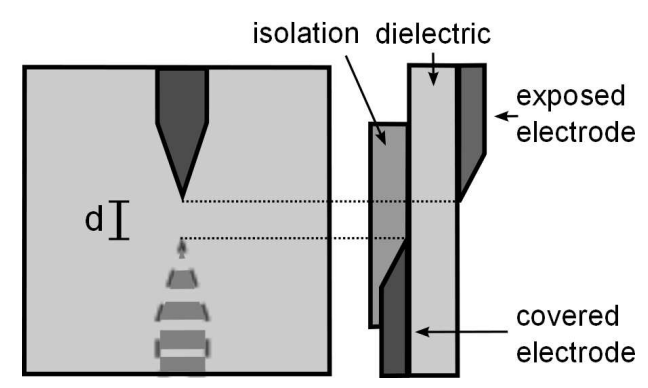

Fig. 1. Configuration of the electrodes in the SBD arrangement (left side: top view, right side: side view).

trode). In this configuration time-consuming CCS studies could be performed on the most intensive emission, namely the $0-0$ vibrational transition of the molecular band of the second positive system (SPS) of nitrogen, since it granted the required time stability of well-localized MDs [13]. Despite several efforts and in particular in case of over voltage operation the time stability was not sufficient to measure the spatio-temporal development of the much weaker molecular bands of the first negative system (FNS) of nitrogen, which give information on the development of ionizing fronts and plasma parameters such as the reduced electric field strength and the electron density. We suppose that the local generation of MDs at one position leads to modification of the surface of the $\mathrm{Al}_{2} \mathrm{O}_{3}$ ceramic and/or the electrode which affects the MDs localisation. The electrode configuration was set in a Plexiglas chamber flushed with dry air. The power supply generated a sinusoidal voltage of several $\mathrm{kV}_{\mathrm{pp}}$ at a frequency of about $60 \mathrm{kHz}$. The voltage was applied to the covered electrode while the exposed electrode was grounded. The voltage was measured with a high voltage probe (Tektronix P6015A), while the current was measured with a Rogowski coil (Tektronix TEK CT-1) surrounding the connection to ground of the exposed electrode. The signals were recorded with a digital storage oscilloscope (Tektronix DPO 4104).

The MDs were investigated by means of short exposure time photography (gated iCCD camera) and CCS. The iCCD camera (PCO Imaging DiCam PRO) was equipped with a far field microscope (Questar QM 100) to enable spatial resolution of $4 \mu \mathrm{m}$. The iCCD photos were taken from top view of the electrode arrangement. The light of the discharge could be projected by a UV achromatic lens to the entrance slit of a monochromator (ACR SpectraPro 500i) with a photomultiplier (PMT, Hamamatsu PMC100-4) behind one of the exit slits. This PMT, operating in single photon mode with maximal gain, records the spatially and spectrally resolved MAIN-signal for the CCS procedure (see Fig 2). This signal was taken from top view as well. To realize the spatially resolved measurements the discharge cell was moved. The maximum axial resolution was $0.05 \mathrm{~mm}$, while no radial structure was resolved in these studies. A second PMT operating at low gain serves for the detection of the SYNC-signal. This signal was recorded from side view. Both pulses are processed by the time-correlated single photon counting (TC-SPC) mod-

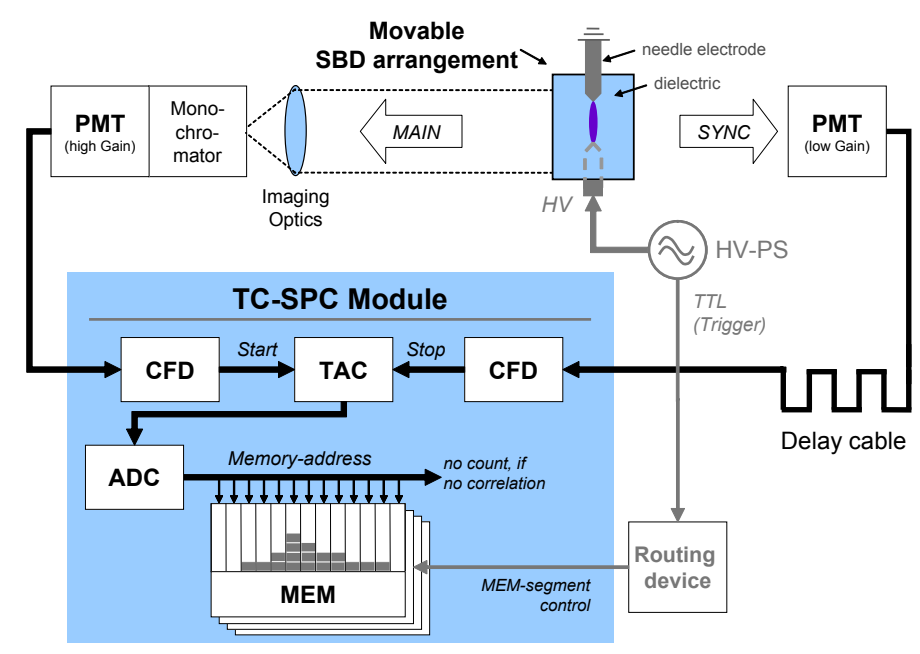

Fig. 2. Scheme of the CCS measurements (details and abbreviations see text).

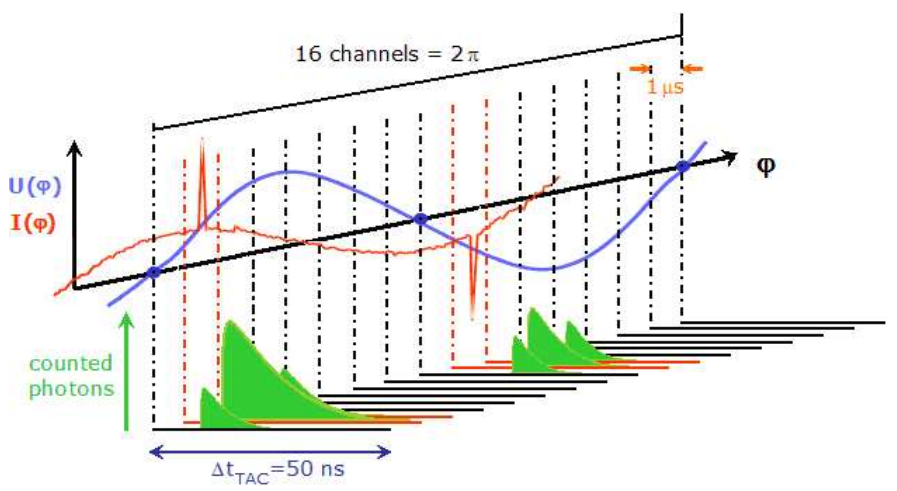

Fig. 3. Scheme of the division of the applied voltage into 16 phase channels for a selected wavelength and spatial position of the CCS measurement.

ule ("Simple Tau" /SPC-150 by Becker and Hickl GmbH), i.e. discrimination and utilization by means of constantfraction discriminators (CFD). Finally the time delay between both pulses is measured by a time-to-amplitude converter (TAC) which gives the relative time-information of the photon within the light pulse of the erratic appearing MD. This information is converted to a memory address by a analogue-to-digital converter (ADC). If no SYNCpulse appears after a MAIN-pulse in the time window of the TAC (typical $50 \mathrm{~ns}$ ) the photon will not be stored in the memory (MEM) ("no count if no correlation").

The phase resolution is realized by controlling the memory segments of the TC-SPC device by the applied voltage. Therefore a TTL signal of the high voltage power supply (HVPS) starts the generation of a time-pattern by means of a routing device (DDG - digital delay and pattern generator from Becker and Hickl). The routing procedure provides a course time scale, which is synchronized with the phase $\varphi$ of the applied voltage. The cycle duration of about $T=1 / 60 \mathrm{kHz} \approx 17 \mu \mathrm{s}$ was divided into 16 intervals or phase channels $(\Delta t$ DDG $=T / 16=1.07 \mu \mathrm{s})$. 


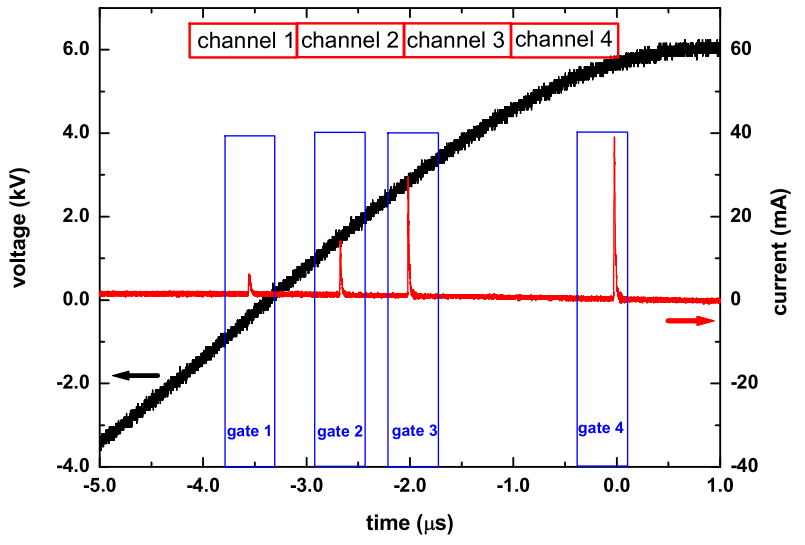

Fig. 4. Exemplary current voltage diagram with the gating of the iCCD camera and the channels of the CCS.

This is demonstrated in Fig. 3, where a typical current slope with one MD per half period is compared with the corresponding result of phase-resolved photon accumulation. In both half periods in three phase-channels photons are registerd, since MDs appear distributed within the rising slope of the applied voltage.

\section{Results and Discussion}

\subsection{General discharge behavior}

Fig. 4 shows exemplary voltage and current curves at a voltage amplitude significantly higher than the burning voltage $\left(12.0 \mathrm{kV}_{\mathrm{pp}}\right.$, so-called overvoltage operation). The voltage is given for the exposed electrode. In general four to six individual current pulses are detectable. Fig. 4 shows an example with four pulses. Within the rising slope of the applied voltage, a tendency to higher current amplitudes is observed. The first MD occurs before the zero transition of the applied voltage due to the negative residual charge accumulated in the previous negative half period. This residual charge results in an electric field superimposing the applied electric field causing the breakdown. The transferred charge of single MDs was up to $1.5 \mathrm{nC}$. In the same manner as for the amplitude of the current pulses, there is a tendency of higher transferred charges within the rising slope of the applied voltage.

In Fig. 5, the general behavior of subsequent MDs in one half period is shown as observed by iCCD imaging. Images (a) to (d) correspond to the gates 1 to 4 indicated in Fig. 4. The first MD (Fig. 5 (a)) develops on the direct path between the electrode positions. Then, the second MD (b) spares the region of the first MD and therefore takes a longer, curved path. The same results are obtained for the third (c) and fourth MD (d). Each new MD evades the regions of previous MDs. If the regions of previous MDs are considered as "forbidden zones", it still takes the shortest way possible. Surprisingly, discharge patterns emerge quite regular as shown in image 4 (e), which is the result of an accumulation over 100 applied voltage
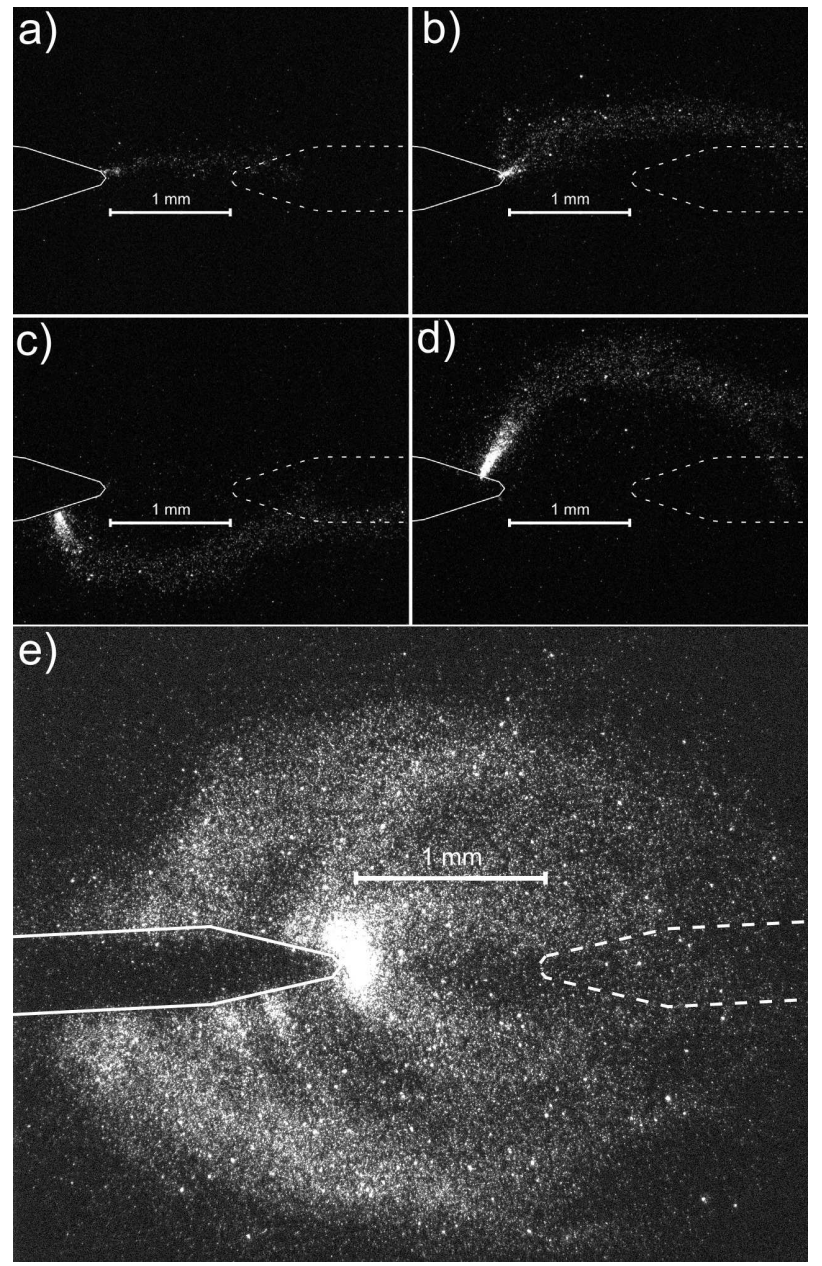

Fig. 5. iCCD images (topview; anode left, cathode right) of subsequent current pulses. Single shots of the first four current pulses (a) to (d) and integrated picture of the whole positive half period over 100 voltage cycles.

cycles. Similar patterns were also investigated at reduced voltages closed to burning voltage with less (two or three) MD channels [13].

The pattern formation is caused by surface effects, in particular, charging of the dielectric. During the first MD the dielectric is positively charged. Similar phenomena are known from volume barrier discharges as well as different coplanar and SBD arrangements $[10,14-18]$. The residual charges weaken the electric field locally and thus, the next breakdown will not occur at the same position as long as the electric field outside this zone is higher. With the rising of the applied voltage, this condition is fulfilled in the adjacent regions. Consequently the MDs appear in a different region and take other paths.

\subsection{MDs at burning voltage operation}

At values of the applied voltage amplitude around the burning voltage (i.e. $4.5 \mathrm{kV}_{\mathrm{pp}}$ ), only one or seldom two MDs in each half period appear. The development of these 
a)

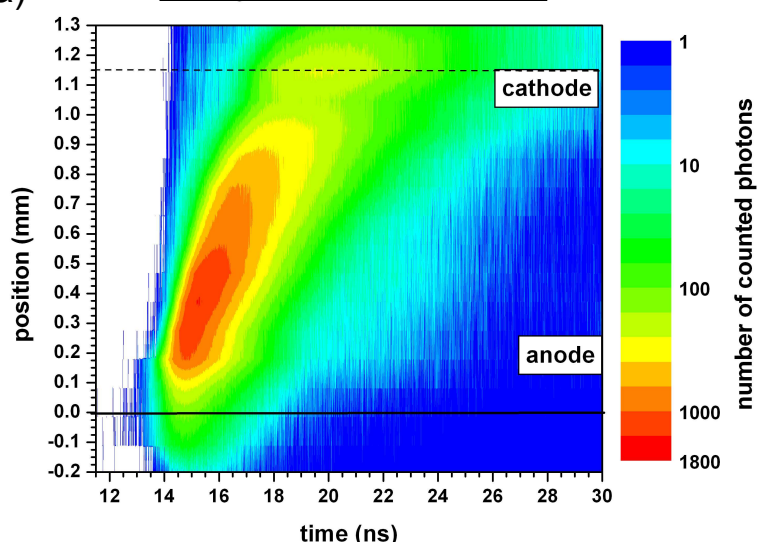

b)



Fig. 6. CCS images of two subsequent channels for burning voltage operation $\left(\mathrm{U}=4.5 \mathrm{kV}_{\mathrm{pp}}\right)$.

MDs was investigated by the measurement of the spatiotemporal distribution of radiation intensity of the $0-0$ vibrational transition of the SPS $(\lambda=337.1 \mathrm{~nm}$; about $11 \mathrm{eV}$ excitation energy by direct electron impact) by means of CCS. The chosen molecular band is the most intensive in the optical emission spectrum. Its luminosity development represents the distribution of the convolution of the reduced electric field $\mathrm{E} / \mathrm{n}$ with the electron density $\mathrm{n}_{e}$. The pictures in Fig. 6 show the development of the SPS-luminosity in the subsequent phase channels 1 (a) and 2 (b), respectively.

The first emission of light appears at the tip of the exposed electrode (see Fig. 6), which serves as the anode (positioned at $0 \mathrm{~mm}$ ), followed by a discharge channel propagating in the direction of the covered cathode (position at $1.15 \mathrm{~mm}$ ). The overall development shows similarities with MDs in VBDs and CBDs [3], since a pre-phase, a cathode directed ionization front and a decay phase are observed. In channel 1 (Fig. 6 a) the maximum velocity of the light emission is about $3.4 \times 10^{5} \mathrm{~m} / \mathrm{s}$. The discharge front propagates slower than in VBD or CBD and decreases on the way to the cathode. The decrease of the discharge front velocity might be explained by the configuration of the initial electric field which is characterized by a decreasing potential in the cathode region [3,13]. However, surface processes and effects caused by residual charges need to be taken into account, too [19]. In the second phase channel (Fig. 6 b), the discharge activity is lower, i.e. less MDs are generated in this time window. Therefore, the maximum number of counted photons is about one order of magnitude lower (1800 vs. 220 photons). This is agreement with the current pulse measurements. In the mean, only at each tenth voltage cycle a second MD per positive half period is observed. From the iCCD photography (Fig. 5) one can suggest that the second MD propagates on a different and longer pathway. The CCS recording in Fig. 6 (b) was performed along the inter-electrode axis without any resolution in the radial direction. Thus, the curved shape of the second MD path is projected on the inter-electrode axis. In such a case, the velocity of the discharge front can not be determined properly from the plot. Furthermore, the signal in Fig. 6 b) may contain photons from first MDs (if they appear later), too. Comparing both images and considering the above mentioned facts for the second MD a similar mechanism as for the first MD can be concluded. The same subsequent phases are observed and the discharge front propagates with a decreasing velocity. Thus except the different position and path the same MDs are generated within the same half period. This behavior is in agreement with investigations on VBDs.

\subsection{MDs at overvoltage operation}

In Fig. 7, the results of the CCS measurement of the SPS for the first four subsequent phase channels are shown for overvoltage operation $\left(11.4 \mathrm{kV}_{\mathrm{pp}}\right)$ of the SBD. The four phase channels cover the first $4 \mu s$ of the half period with positive polarity (see Fig. 4). The CCS measurements were focused on the anode region and therefore don't show the complete discharge gap. The overall development in the first time channel (Fig. 7 a) shows the pre-phase and the cathode directed ionization front. It develops with a velocity of $3.8 \times 10^{5} \mathrm{~m} / \mathrm{s}$. In contradiction to the single-MD mode behind the ionization front a second maximum of light emission is observable at the anode tip. In the following phase channels (Fig. 7 b-d), the first maximum of emission (which is correlated with the head of the ionization front) is shifted towards the cathode. The velocity of the first MD increases from channel to channel from $3.8 \times 10^{5} \mathrm{~m} / \mathrm{s}$ to $5.4 \times 10^{5} \mathrm{~m} / \mathrm{s}$ (channel 2), $6.5 \times 10^{5} \mathrm{~m} / \mathrm{s}$ (channel 3) and $7.7 \times 10^{5} \mathrm{~m} / \mathrm{s}$ (channel 4). The anode light emission increases in the same manner and shows a cathode directed propagation, too. The velocity of this phenomenon also increases with similar rate (from $1.6 \times 10^{5} \mathrm{~m} / \mathrm{s}$ to $2.4 \times 10^{5} \mathrm{~m} / \mathrm{s}$ to $2.8 \times 10^{5} \mathrm{~m} / \mathrm{s}$ ). It has to be mentioned that the actual velocity must be higher than determined from the plots in Fig. 7 due to the curved shapes and thus longer pathways of MDs (see Fig. 5).

The interpretation of the investigated phase dependence is rather difficult since photons from different MDs may overlap in one phase channel. In general an increase 
a)

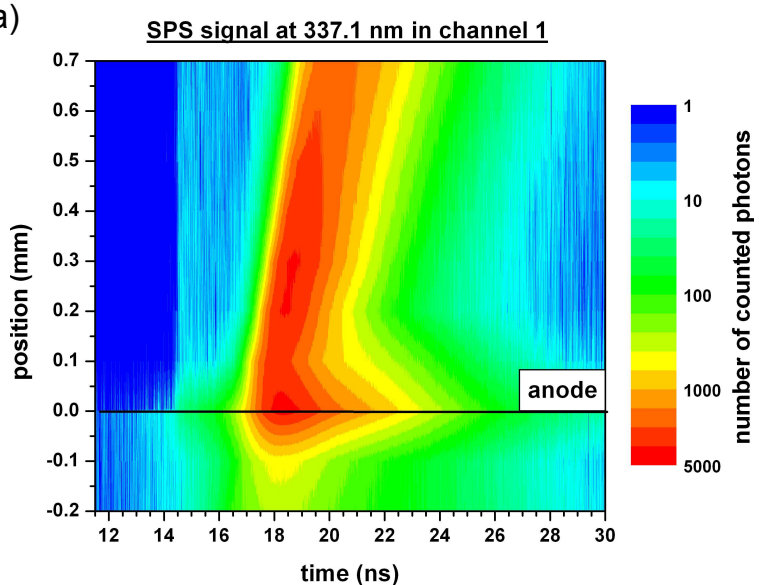

C)

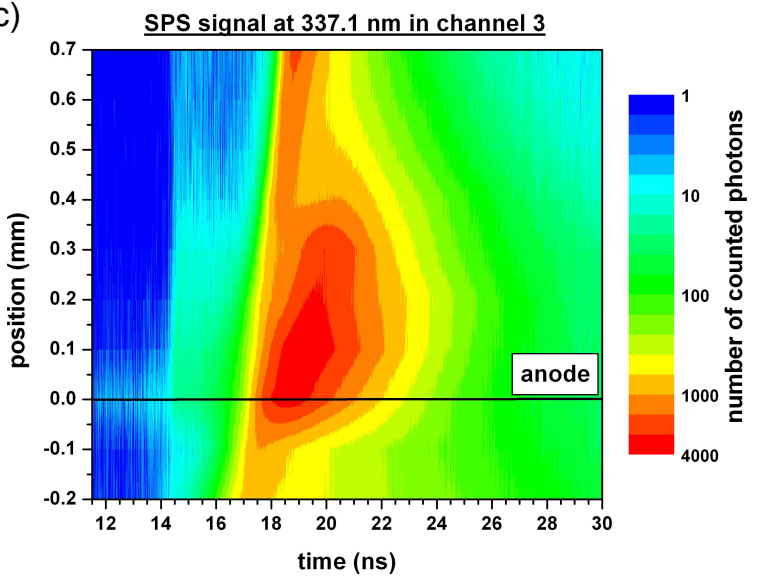

b)

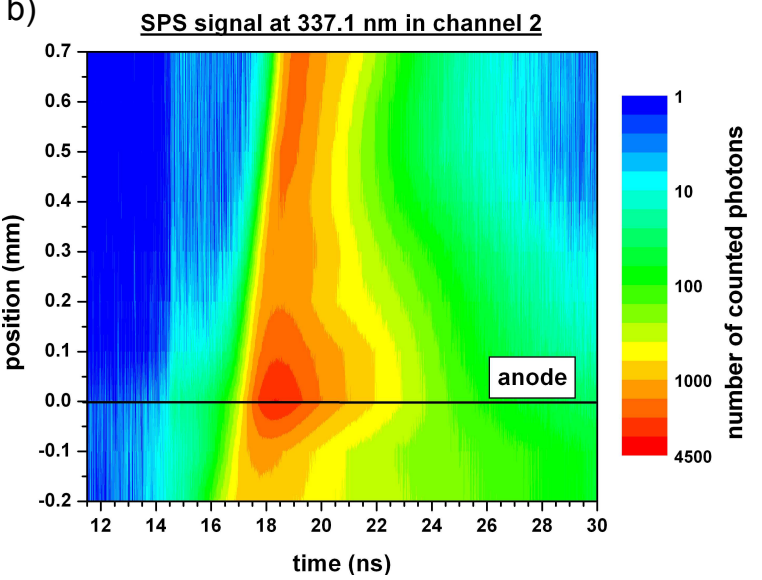

d)

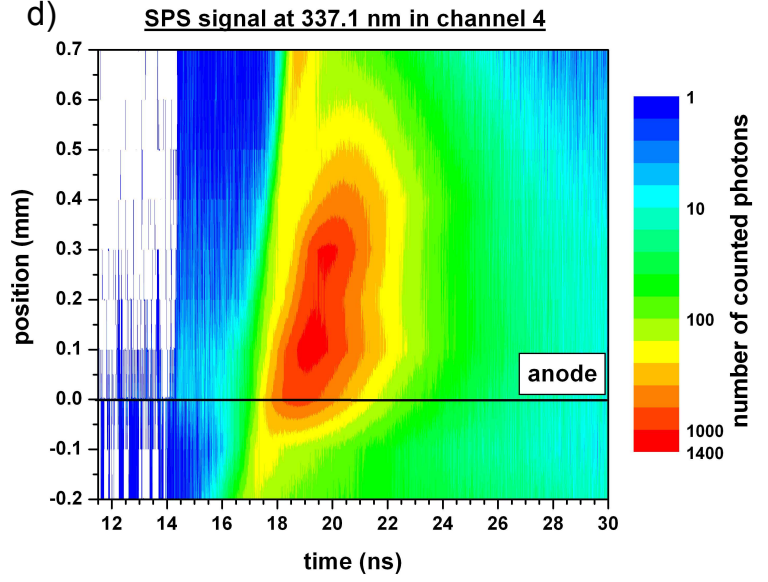

Fig. 7. Spatio-temporal development of MDs in four subsequent channels at over voltage operation $(\mathrm{U}=11.4 \mathrm{kV} p p)$.

of the second emission event at the anode with applied voltage rise from channel 1 to channel 4 (see Fig. 4) is observed. The phenomenon can be compared with the socalled anode glow, which is well known for VBDs and CBDs $[6,20,21]$. Behind the cathode directed ionization front electrons are drifting towards the anode. In this region the electric field increases again and electrons can gain further energy on their way to the anode. At higher voltage amplitude the electric field in front of the needle electrode reaches a value high enough for sufficient excitation of nitrogen molecules. It seems that the MD development in this SBD arrangement becomes more similar to that one in VBD geometries due to the increased applied voltage.

\section{Conclusion and Outlook}

In this contribution, subsequent MDs in an asymmetric needle-needle SBD arrangement in the positive half period of a sinusoidal driving voltage were investigated by short exposure time iCCD photography and CCS diagnostic. The SBD arrangement was operated in two different modes. In the first one, the applied voltage amplitude is close to the burning voltage, which results in only one or two MDs per half period. The second mode is characterized by the generation of up to six MDs per half period which is caused by overvoltage. All MDs propagate directly on the dielectric surface.

In each mode the first MD propagates on the direct path between the pin-electrode positions. The local charging of the dielectric guides the following MDs in a different region of the electrode area. This leads to the formation of regular surface discharge patterns. At burning voltage operation very similar MDs are generated within the same half period. These MDs are characterized by the development of a cathode directed ionization front with decreasing velocity. The results confirm, that the residual surface charges determine the spatial distribution of MDs. Increasing the applied voltage amplitude, the number as well as the properties of the MDs are changed significantly. In particular faster ionization fronts are investigated at higher values of the applied voltage corresponding with longer MD channels. Due to the higher electric field in front of the anode electrons gain energy behind the volume space charge produced by the cathode directed ionization front. This leads to a second maximum of light emission at the anode, similar as observed for VBDs and 
CBDs, but not for the SBD so far. It increases during the continuing applied voltage rise within the half period. Obviously the higher amplitude of the applied electric field and the local charging result in longer MD channels with faster ionization fronts, higher electron densities and thus larger discharge currents.

\section{Acknowledgment}

The authors are grateful to Wolfgang Reich for technical assistance and Dr. Ralf Basner and Dr. Manfred Kettlitz for helpful remarks and fruitful discussions. The work was partly supported by the German Federal Ministry of Education and Research (BMBF, project 03IO1072).

\section{References}

1. K.H. Becker, U. Kogelschatz, K.H. Schoenbach, R.J. Parker (eds.) Non-Equilibrium Air Plasmas At Atmospheric Pressure (Institute Of Physics Publishing, New York 2004)

2. V.I. Gibalov, G.J. Pietsch, J. Phys. D: Appl. Phys. 33 (2000) 2618-2636, DOI: 10.1088/0022-3727/33/20/315

3. H. Grosch, T. Hoder, K.-D Weltmann, R. Brandenburg, Eur. Phys. Jour. D (2010) DOI: 10.1140/epjd/e2010-00239-8

4. H.-E. Wagner, K.V. Kozlov, R. Brandenburg et al., Low temperature plasmas edited by $\mathrm{R}$. Hippler, H. Kersten, M. Schmidt and K.-H. Schoenbach (Wiley-VCH, Weinheim 2008) 2nd edition, Vol. 1 271-306

5. W. Becker, Advanced time-correlated single photon counting techniques (Springer-Verlag, Berlin 2005)

6. K.V. Kozlov, H.-E. Wagner, R. Brandenburg, P. Michel, Phys. D: Appl. Phys. 34 (2001) 3164-3176, DOI: 10.1088/0022-3727/34/21/309

7. R. Brandenburg, T. Hoder, H.-E. Wagner, IEEE Trans. Plasma Sci. 36 (2008) 1318-1319, DOI: 10.1109/94.407017

8. T. Hoder, M. Sira, K.V. Kozlov, H.-E. Wagner, Contrib. Plas. Phys. 49 (2009) 381-387, DOI: 10.1088/00223727/42/4/049802

9. S. Celestin, G. Canes-Boussard, O. Guaitella, A. Bourdon, A. Rousseau, J. Phys. D: Appl. Phys. 41 (2008) 205214 10pp, DOI: $10.1088 / 0022-3727 / 41 / 20 / 205214$

10. T. Hoder, R. Brandenburg, R. Basner, K.-D. Weltmann, K.V. Kozlov, H.-E. Wagner, J. Phys. D: Appl. Phys. 43 (2010) 8pp, DOI: 10.1088/0022-3727/43/12/124009

11. D. F. Opaits, M. N. Shneider, Richard B. Miles, A. V. Likhanskii, S. O. Macheret, Phys. Plasmas 15 (2008) 073505 , DOI: $10.1063 / 1.2955767$

12. J. Deng, S. Matsuoka, A. Kumada, K. Hidaka, J. Phys. D: Appl. Phys. 43 (2010) 495203 8pp, DOI: 10.1088/0022$3727 / 43 / 49 / 495203$

13. R. Brandenburg, H. Grosch, T. Hoder, K.-D. Weltmann, Proc. of 37th Conference on Plasma Physics of the European Physical Society (EPS), Dublin/Ireland 2010.

14. A. Kumada, S. Okabe, K. Hidaka, J. Phys. D: Appl. Phys. 42 (2009) 095209 (8pp), DOI: 10.1088/0022$3727 / 42 / 9 / 095209$

15. Y. Zhu, T. Takada, K. Sakai, D. Tu, J. Phys. D: Appl. Phys. 29 (1996) 28922900, DOI: 10.1088/0022$3727 / 29 / 11 / 024$
16. J.P. Boeuf, Y. Lagmich, L.C. Pitchford, J. Appl. Phys. 106 (2009) 023115, DOI: 10.1063/1.3183960

17. K. Allegraud, O. Guaitella, A. Rousseau, J. Phys. D: Appl. Phys. 40 (2007) 7698-7706, DOI: 10.1088/00223727/40/24/017

18. Yu. V. Yurgelenas, H.-E. Wagner, J. Phys.D: Appl. Phys. 39 (2006) 4031-4043, DOI: 10.1088/0022-3727/39/18/015

19. L. Niemeyer, IEEE Trans. Dielect.El. In. 4 (1995) 510-528, DOI: $10.1109 / 94.407017$

20. R. Brandenburg, H.-E. Wagner, A.M. Morozov, K.V. Kozlov, J. Phys.D: Appl. Phys. 38 (2005) 1649-1657, DOI: 10.1088/0022-3727/38/11/003

21. T. Hoder, M. Sira, K.V. Kozlov, H.-E. Wagner, J. Phys. D: Appl. Phys. 41 (2008) 9pp, DOI: 10.1088/0022$3727 / 41 / 3 / 035212$ 